\title{
Linee guida della Società Svizzera di Psichiatria Assicurativa, nel campo delle perizie mediche che riguardano i disturbi psichici*
}

\author{
Società Svizzera di Psichiatria Assicurativa, Associazione svizzera di medici esperti in questioni assicurative, \\ in relazione con i disturbi psichici e psicosomatici
}

Deutsch erschienen

in Nr. 20/2004

La version française

a paru dans le $n^{\circ} 36 / 2004$
* Testo approvato e messo in vigore dall'Assemblea generale della Società Svizzera di Psichiatria Assicurativa, il 13 novembre 2003.

1 Agency for Health-Care Policy and Research. Istituzione americana che elabora le «Clinical Practice Guidelines».

\section{Introduzione}

1. Definizione del concetto di «linee guida» L'AHCR [1] definisce il concetto di linea direttiva nel modo seguente: Le linee guida sono proposte e raccomandazioni sviluppate in modo sistematico, allo scopo di aiutare pazienti e medici a prendere, in situazioni cliniche precise, decisioni utili dal punto di vista della prevenzione, della diagnosi, del trattamento, del decorso e delle conseguenze delle malattie. Riflettono lo stato delle conoscenze ad un dato momento, e devono essere riviste periodicamente. Tengono conto dei risultati di studi controllati; l'influenza della soggettività dei periti si riduce ad un minimo grazie al ricorso a tecniche quali le conferenze di consenso, le procedure Delphi, o il metodo del gruppo nominale.

\section{Ruolo delle linee guida}

Le presenti linee guida rappresentano delle indicazioni pratiche per l'attività del perito. Si basano su un esame dei dati scientificamente accertati, e tengono conto delle esperienze e delle necessità della pratica. La loro formulazione si basa su d'un ampio consenso tra periti. Hanno il carattere di raccomandazioni, non quello di una norma legale inderogabile.

\section{Campo di validità delle linee guida}

Queste linee guida riguardano le perizie nel campo assicurativo, nei casi di problemi d'ordine psichiatrico, psicosomatico, e psicoterapeutico.

\section{Definizione della perizia medica}

Una perizia è definita, ai sensi delle presenti linee guida, come presa di posizione scritta di un esperto psichiatra indipendente, nell'ambito di un mandato. In questo referto, l'esperto risponde a domande riguardanti la psichiatria assicurativa.

\section{Principi}

\section{Disturbi psichici}

I disturbi psichici comportano aspetti biologici, intrapsichici, e sociali (quest'ultima dimensione include anche considerazioni d'ordine culturale e sistemico). Per questo oggi parliamo di un modello bio-psico-sociale della psichiatria. È compito del perito tenere conto di tutti questi differenti aspetti della situazione sottopostagli, che deve anche descrivere nel suo rapporto.

\section{Esigenze giuridiche}

Affinché un rapporto medico abbia un valore probante, secondo una giurisprudenza costante del TFA, è indispensabile che il rapporto peritale rispetti le esigenze qualitative seguenti. I punti importanti in discussione devono essere oggetto di uno studio approfondito. Il rapporto si deve fondare su esami completi. L'esperto deve aver preso in considerazione anche i sintomi lamentati dalla persona esaminata. Il rapporto dev'essere stato redatto con la piena conoscenza degli incarti precedenti. La descrizione delle correlazioni e delle conseguenze del quadro medico, e la loro valutazione, devono essere plausibili. Infine, anche le conclusioni del perito devono essere debitamente motivate.

\section{La perizia psichiatrica nel contesto del diritto assicurativo}

La perizia psichiatrica consiste nella presentazione clinica di un caso dal punto di vista psichiatrico. Il medico qui deve assumere il ruolo di un esperto, la cui funzione è di aiutare l'amministrazione o la giustizia in un procedimento di presa di decisione. La risposta alle domande d'ordine giuridico che gli sono poste esige una certa familiarità con il modo di pensare che esse sottendono. La pratica del diritto presuppone un modo di pensare normativo, cui il medico sarà 
tenuto ad adattarsi al momento in cui riceverà un incarico come perito. La scienza medica si basa però piuttosto su un approccio empirico, che non su di un approccio normativo. La medicina assicurativa rimane una parte della medicina clinica, cui egli deve comunque conformarsi. Deve presentare sia il risultato delle sue investigazioni, sia la sua valutazione della situazione, in un modo adeguato a fornire all'istanza che l'ha incaricato (generalmente un'assicurazione o un tribunale) le informazioni di cui ha bisogno per far propria una decisione. Le differenze tra l'epistemologia medica e quella giuridica richiedono un procedimento che traduca le constatazioni mediche, in modo da adattarle al quadro proprio della riflessione giuridica. Il fatto che il mandatario si aspetti delle risposte chiare, formulate nei termini delle sue categorie di riflessione, non deve, all'occorrenza, indurre il perito ad assumere posizioni che non siano più conformi alle esigenze della scienza medica. Mettere in luce i limiti delle conoscenze mediche, e rispondere solo in parte, se del caso, o addirittura non rispondere alle domande poste, rimane un compito primordiale della medicina assicurativa. Essa dunque deve coltivare un dialogo costante con gli assicuratori e con i giuristi, prestando attenzione a mantenere le condizioni che le permettano un esercizio corretto del «processo di traduzione». Si tratta d'insistere affinché le domande sottoposte siano pertinenti, e di rifiutarsi ad assumere la responsabilità di rispondere a domande non mediche, o pseudomediche.

La psichiatria, proprio perché è una scienza medica, ha i suoi limiti, ed è importante che il perito li sottolinei, quando si trova confrontato a domande alle quali è impossibile rispondere in modo sufficientemente chiaro e preciso.

\section{Scopo della perizia psichiatrica}

La perizia psichiatrica ha per scopo di verificare un eventuale danno alla salute, dal punto di vista psichiatrico, in una prospettiva medica globale. A seconda delle circostanze particolari del mandato, e della natura delle domande poste, si tratterà di valutare le ripercussioni sulla capacità di lavoro della persona esaminata, di esprimere proposte per il proseguimento della cura, e per eventuali misure di riabilitazione, di valutare un danno permanente nell'ottica del diritto ad una rendita, o ad un'indennità per danno all'integrità, o ancora, di dare al giurista gli elementi necessari per una decisione in materia di causalità.

\section{Obiettività e neutralità dell'esperto}

Il perito deve assumere una posizione imparziale e neutra tra le assicurazioni e il peritando. Ma per quel tipo di dialogo indispensabile per raccogliere le informazioni necessarie con la persona da esaminare, è necessario stabilire con quest'ultima una relazione empatica. Sarebbe dunque meglio parlare di «parzialità multidirezionale», per descrivere l'atteggiamento del perito. Dovrebbe restare, peraltro, obiettivo, nei limiti in cui questo è possibile nella psichiatria, sforzandosi costantemente di prendere coscienza e di distanziarsi delle concezioni e dalle motivazioni, che riguardano la sua stessa storia e la sua stessa persona. Il colloquio clinico costituisce il principale metodo d'investigazione dello psichiatra. Si tratta, per definizione, di un processo dialogico il cui risultato non è determinato solo dal peritando, ma anche dal perito. Il risultato dell'esame sarà dunque in ogni caso influenzato dall'esaminatore. La riflessione critica esercitata da questo ultimo sui suoi modi di pensare, di sentire e di comportarsi costituisce un criterio di qualità centrale in una perizia psichiatrica.

\section{Esigenze poste al perito}

Il perito deve aver seguito una formazione completa come specialista in psichiatria e psicoterapia. Una familiarità con la problematica e con i concetti del diritto assicurativo è indispensabile. Il perito deve formarsi ulteriormente in psichiatria, in particolare nella medicina assicurativa. Dovrebbe condividere con dei colleghi una riflessione a proposito della sua pratica, nel quadro d'un circolo di qualità, o d'un gruppo d'intervisione e supervisione. Si deve anche interessare a questioni d'ordine culturale e sociale, che in particolare riguardino il mondo del lavoro ed il sistema assicurativo.

\section{II procedimento peritale}

\section{L'attuazione della perizia}

Dapprima, il perito, raccoglie le informazioni pertinenti, iniziando dallo studio dell'incarto, dalle informazioni fornite dalla persona esaminata, da quelle fornite eventualmente da terzi, dall'esame del quadro clinico, e dal comportamento del soggetto. In un secondo tempo, si sforza di elaborare una rappresentazione globale coerente, a partire da questi differenti materiali.

Nella misura in cui non vi perviene in modo soddisfacente, e non è quindi in grado di rispondere alle domande postegli, il perito si sforza nel modo migliore possibile di completare e di affinare la sua rappresentazione della situazione, effettuando o chiedendo di effettuare investigazioni complementari, o approfondendo la sua riflessione. 
Appare evidente che possono notevolmente variare sia la durata della preparazione di un rapporto peritale, sia il suo numero di pagine. Il lavoro del perito consiste essenzialmente nell'integrare i diversi aspetti, che vanno poi riassunti nella scrittura del testo.

\section{Perizia psichiatrica e processo interpersonale}

Quando assume la funzione d'esperto, il medico rimane medico, e resta, perché tale, sottomesso al principio fondamentale del «primum nihil nocere». Sottoporre qualcuno ad una perizia avrà inevitabilmente delle ripercussioni sullo stato mentale della persona esaminata. Qualunque possano essere gli sforzi d'obiettività e di neutralità dell'esaminatore, ogni perizia medica rappresenta un intervento suscettibile d'influire sull'evoluzione della malattia.

È di conseguenza indispensabile che il perito tenga conto, nella sua riflessione, della natura dell'interazione emotiva tra il peritando e se stesso, dei fattori motivazionali del peritando, e dei problemi collegati ai meccanismi di difesa, dal transfert al contro-transfert.

\section{I differenti livelli di valutazione della qualità di una perizia}

Un primo livello di valutazione riguarda la qualità della raccolta e della presentazione delle informazioni. Ad un livello superiore, si apprezzeranno la qualità dell'integrazione delle informazioni, collegate in un quadro globale coerente. In seguito, viene l'aspetto convincente e completo della discussione, ed infine, la chiarezza e la precisione delle risposte alle domande poste. Se restano aperte delle domande, o se delle contraddizioni non hanno potuto trovare una soluzione, il perito lo dovrebbe menzionare esplicitamente.

\section{Fattori esterni}

Ci sono dei fattori, indipendenti dal perito, che possono influenzare la qualità di una perizia:

- qualità, attualità e completezza della documentazione a disposizione (comprende delle informazioni sulla natura e sulle esigenze del posto di lavoro, e sul livello dell'adattamento professionale cui il peritando ha dato prova fino a quel momento?);

- circostanze e motivi della richiesta di perizia;

- qualità delle domande poste;

- qualità della collaborazione nel triangolo, rappresentato dall'assicurazione, dall'avvocato della persona da peritare, e dal perito;

- livello d'informazione, di motivazione, e di cooperazione del peritando;
- qualità della collaborazione dei familiari della persona esaminata, dei suoi medici curanti, del suo datore di lavoro, ecc.

\section{Parte pratica}

\section{Organizzazione ed attrezzatura dell'ambulatorio medico, rispettivamente dell'istituzione}

I locali devono permettere lo svolgimento dell'esame, in una cornice tranquilla e gradevole. Gli appuntamenti devono essere comunicati in modo chiaro, ed anche il perito li deve rispettare puntualmente.

\section{Studio degli incarti}

Una buona conoscenza dell'incarto è importante per la valutazione, poiché permette al perito di rappresentarsi l'evoluzione diacronica della situazione, e di fare il punto dei pareri già espressi, che poi dovrà commentare nella sua discussione.

\section{Informazioni da dare alla persona esaminata}

Il peritando deve essere informato chiaramente fin dal primo momento che l'esame si svolge nel quadro di una perizia medica. Deve sapere che il perito ha l'intenzione di farsi un parere indipendente. Deve anche sapere che ha il dovere di rispondere alle domande che gli sono poste. Il perito, inoltre, è sciolto dal segreto professionale nei confronti dell'istanza che gli ha dato l'incarico. Il peritando, per quanto possibile, dovrebbe essere informato in un modo appropriato circa il risultato della perizia.

\section{Esame peritale}

Il peritando deve essere esaminato personalmente dal perito. L'esame clinico, che comprende la presa dell'anamnesi, l'esame semiologico e l'osservazione del comportamento, costituisce la parte centrale della perizia. L'evoluzione della relazione interpersonale deve essere oggetto di un'attenzione particolare (cfr. sopra, al punto III.2.).

L'ampiezza e la profondità delle investigazioni saranno funzione delle particolarità del caso e della natura delle domande poste.

Come già menzionato al punto III.2., la qualità del procedimento peritale dipende anche dalla motivazione e dalla collaborazione della persona esaminata. Hanno un carattere determinante, oltre alle dichiarazioni del peritando, il quadro clinico, il comportamento osservato, e il confronto con le informazioni obiettive che si trovano nell'incarto. 


\section{Relazione con la valutazione somatica}

La valutazione degli aspetti somatici del quadro precede generalmente nel tempo la valutazione psichiatrica. È particolarmente importante disporre di un bilancio somatico di buona qualità, se ci si deve pronunciare nei casi di disturbi psicosomatici, perché parecchie diagnosi del capitolo F dell'ICD-10 possono essere poste solamente dopo avere escluso un'origine somatica dei sintomi.

L'esame somatico e l'esame psichiatrico dovrebbero essere praticati in modo separato, dai rispettivi specialisti. Non è raro, tuttavia, che sia il dialogo interdisciplinare a permettere di chiarire nel modo migliore il significato di un quadro clinico particolare.

\section{Informazioni riferite da terzi}

Può essere importante sapere come la persona esaminata sia percepita nel suo ambiente familiare e professionale. Ma queste informazioni di terzi possono essere raccolte solo con l'autorizzazione del peritando. Un contatto con il medico curante (medico generico o psichiatra) può portare preziose informazioni sugli antecedenti medici, sulla personalità del peritando, sulla sua collaborazione nel quadro della cura, sulla qualità del suo ambiente. Può anche essere utile nell'elaborazione di proposte terapeutiche. Alcune informazioni riferite da terzi possono così costituire una parte importante della perizia, ma non vi si devono obbligatoriamente ritrovare. Le persone interrogate devono essere informate sulla natura del colloquio, in analogia a quanto menzionato al punto IV.3.

\section{Esami complementari}

Dei test psicologici possono rappresentare un supplemento all'investigazione. L'esame clinico e la sua messa in parallelo con l'anamnesi mantengono tuttavia un carattere determinante.

Anche una documentazione standardizzata della semiologia psichiatrica secondo certe scale particolari, come per esempio il sistema AMDP, può essere considerata al massimo come un supplemento dell'esame clinico.

Degli esami di laboratorio, ed altri esami paraclinici si possono rivelare indicati, almeno in certi casi.

\section{Redazione del rapporto di perizia}

Il rapporto di perizia deve essere strutturato chiaramente. La fonte delle notizie che vi figurano deve essere indicata. Deve essere redatto in un linguaggio chiaro e comprensibile al giurista.

Il rapporto non deve essere più lungo del necessario. In un'ottica di coerenza e di leggibilità, si eviteranno le descrizioni troppo dettagliate, e le ridondanze.

Deve comprendere i seguenti capitoli, eventualmente suddivisi in sezioni:

- introduzione;

- anamnesi ed informazioni tratte dall'incarto;

- indicazioni soggettive del peritando;

- osservazione ed altri risultati di esami;

- discussione e risposta alle domande.

\section{Discussione}

La discussione si fonda sull'integrazione delle informazioni provenienti dall'incarto, dall'anamnesi, dalle indicazioni soggettive, dall'osservazione clinica, e dal risultato degli esami complementari, eventualmente effettuati. Il procedimento intellettuale del perito dovrebbe essere presentato in modo comprensibile, e gli eventuali disaccordi rispetto alle valutazioni anteriori dovrebbero essere commentati e giustificati.

Il perito dovrà eventualmente mettere a fuoco e spiegare le discordanze tra le indicazioni del peritando ed il quadro clinico osservato. Dovrà anche menzionare le zone di incertezza, ed i punti contraddittori.

\section{Diagnosi}

Per principio, nella diagnosi dei disturbi psichici, si utilizzeranno le classificazioni ICD-10 o DSMIV. La diagnosi sarà se possibile completata da riflessioni d'ordine eziologico, o da altre precisazioni.

Inoltre, altri punti importanti sono la diagnosi di personalità, la descrizione della psicodinamica, la valutazione dell'influenza di fattori di natura prettamente sociale sull'evoluzione, e la valutazione del grado di gravità del quadro.

\section{Inabilità (incapacità) lavorativa}

L'incapacità lavorativa non deriva direttamente dalla diagnosi. La si valuta sulla base del tipo e dell'importanza delle limitazioni funzionali causate dai disturbi. Le possibilità di compensazione devono ricevere un'attenzione appropriata.

La capacità lavorativa sarà indicata in percentuale, oppure in ore per giorno. Quando si indica una capacità di lavoro con una modalità oraria, bisogna precisare la natura delle limitazioni addizionali, che possono consistere nella necessità di fare delle pause, in un rendimento ridotto, in una flessibilità diminuita. Anche qui, il perito deve sviluppare le ragioni che motivano le sue conclusioni.

\section{Risposta alle domande peritali}

Il perito, nella discussione, deve tenere conto di tutte le domande che gli sono sottoposte, ma 
deve rispondervi solo nella misura in cui questo gli è possibile, sulla base delle sue conoscenze mediche.

\section{La questione della riabilitazione}

Il successo delle misure di riabilitazione che il perito sarà eventualmente chiamato a proporre non dipende solo dalla loro natura appropriata, ma anche, tra l'altro, dalle competenze e dall'impegno dell'assicuratore (particolarmente da parte del «case manager», responsabile di seguire sistematicamente il caso), dalla motivazione del peritando, e dal sostegno che otterrà dal suo ambiente.

\section{Esame di persone d'altra lingua}

Se il livello di comprensione linguistica tra il perito e la persona esaminata non è sufficiente, si dovrà ricorrere ad un interprete qualificato.

\section{Perizie interdisciplinari}

Una valutazione d'insieme soddisfacente non si ottiene in una giustapposizione di un insieme di perizie elaborate da specialisti delle differenti discipline, ma nel dialogo tra i differenti specialisti implicati. L'importanza e l'ampiezza della parte psichiatrica di una perizia interdisciplinare dipende dalla sua importanza specifica, in quel caso particolare.

\section{Catamnesi}

Ogni attività scientifica richiede a colui che la esercita, di sviluppare le sue conoscenze e di perfezionare la sua pratica, in funzione dei risultati concreti del suo lavoro. È augurabile dunque che i periti ricevano, più spesso di quanto non sia attualmente il caso, un feed-back da parte delle istanze che hanno affidato loro il mandato peritale. 